\title{
Using Travertine in Pervious Pavement to Control Urban-Flooding and Storm Water Quality
}

\author{
Hamidreza Rahimi ${ }^{1}$, Xiaonan Tang ${ }^{2}$, Sadra Rahimi $^{3}$ \& Prateek Kumar Singh ${ }^{1}$ \\ ${ }^{1} \mathrm{PhD}$ candidate of Engineering at University of Liverpool, United Kingdom \\ ${ }^{2}$ Associate Professor of Civil Engineering at Xi'an Jiaotong-Liverpool University, China \\ ${ }^{3}$ Undergraduate student of HSE Engineering at Shahid Beheshti University, Iran \\ Correspondence: Hamidreza Rahimi, PhD candidate of Engineering at University of Liverpool, United Kingdom. \\ E-mail: Hamidreza.rahimi@liverpool.ac.uk
}

Received: May 23, 2018; Accepted: June 20, 2018; Published: June 29, 2018

\begin{abstract}
The conventional methods for controlling urban-flooding are to build raceway networks to transfer flood water away as quickly as possible. However, due to fast increase of urban population, the conventional methods are facing some problems especially when rainfall intensity is higher than design expectation. The main reason for such problems are due to impervious surfaces. Therefore, pervious pavements, such as porous asphalts or pervious concrete blocks, are now recommended to use by environmental engineers. Compared with conventional pavement surfaces, pervious pavements have lots of benefits although they are relatively expensive to build. This paper introduces the mineral rock of Travertine as the materials for pervious pavements, and describes an experimental model to determine the inflation patterns and storm water quality improvement in flood precipitating simulation.

The results indicated that Travertine pavement can not only decrease more than 90\% of Copper, Lead and Zinc but also play an important role in urban-flood management with a 50\% decrease of storm water.
\end{abstract}

Keywords: Pervious Pavement, Pollutants, Travertine, Urban flooding, Water quality

\section{Introduction}

The construction of impervious pavements has been becoming one of the main problems in controlling urban flooding due to the rapid urbanization, which has some negative influence on the urban water cycle. Impervious pavements reduce the runoff infiltration into the ground, so resulting the significant environmental problems. The increased runoff can cause many urban water problems which affects the people's daily life (Acioli, 2005; Gilbert, 2006). On the other hand, the imperviousness causes the runoff which may also cause to the overloading of classic drainage channels, thus forming superficial water courses or causing flooding. Moreover, as runoff wash the streets and move pollution along the pavement surfaces, it can also cause serious water quality problems (Brattebo, 2003; Gomez-Ullate, 2010).

Rapid urbanization needs to be managed in different aspects such as water urban management, which involves rainwater, wastewater and the groundwater management. Recycling rainwater by reusing runoff is now a water management method, which has been used in many development countries. For example, in Australia there are many methods to recycle the runoff. Among those methods, there are some techniques that recycle runoff for nonpotable demands (Hatt et al., 2005). This also happens in Germany, the approaches of recycling runoff from urban surface have been expanded (Nodle, 2007). Based on these kind of methods, many new studies for rainwater reuse have been emerged to make urban drainage more sustainable. Pervious pavement is a type of these techniques to treat infiltration water of rainfall to passive storage water (Castro Fresno, 2005).

Compared with a conventional pavement, a pervious pavement structure has a higher permeability of the surface short period (Jayasuriya et al., 2007). Based on Zahng (2006), porous pavements are a pavement layer with a high infiltration capacity; porous asphalt is a typical example of porous pavement. But permeable pavements are constructed using an impervious materials with cracks and voids between them; pervious concrete blocks are the most famous type of these pavements.

It should be noticed that both porous asphalt and pervious concrete blocks are not economically affordable in many countries such as Iran. Therefore, this paper for the first time introduces a new pavement called Travertine, a mineral and natural pavement, which is classified as a porous pavement and can naturally infiltrate runoff thorough 
its voids. Travertine is a form of limestone deposited by mineral springs, especially hot springs. Travertine often has a fibrous or concentric appearance and exists in white, tan, cream-colored, and even rusty varieties. It is formed by a process of rapid precipitation of calcium carbonate, often at the mouth of a hot spring or in a limestone cave. In this study, Travertine has been under experimental studies for evaluating its ability to control the quality and quantity of storm water runoff. The collected data has been compared to porous asphalt and concrete blocks as most famous and useful types of pervious pavements.

\section{Materials and Methods}

The results of this study are based on experimental data obtained from the FUM (Note 1) water engineering laboratory. The study on quality and quantity of infiltrated water requires the experimental setup which has been built as below.

The experiments on the different types of pavement were conducted in a $2 \mathrm{~m} \times 1 \mathrm{~m} \times 0.6 \mathrm{~m}$ steel box in which the bottom plate with holes was constructed for water to pass through (Figures $1 \& 2$ ). A bottom plate was made of Galvanize, which has a good resistance against corrosion (Figure 2). A rainfall simulator with 8 spaced sprays (Figure 3) was set up above the pavement surface to cover an area of $2 \mathrm{~m} \times 1 \mathrm{~m}$. The rainfall intensities were controlled by a flow meter (Figure 4). The water flowing through the pavement was collected from an inclined steel plate (Figure 5) that is underneath the pavement and connected only to the central $2 \mathrm{~m} \times 1 \mathrm{~m}$ area.

For better simulation it was necessary to use sub-structure materials under the travertine pavement. Sub-structure material selection is an important factor for pavement engineers who construct pervious pavements. The load bearing capacity and hydraulic conductivity of the selected material are important factors that determine the success of the system (Zhang, 2006).

The sub-structure materials that were used in this research were divided to two parts: a base layer and a subbase layer, which are exactly the same as a pavement construction. In our study, the aggregate sizes of base materials and sub base materials are $2.36-4.75 \mathrm{~mm}$ and $4.75-10 \mathrm{~mm}$, respectively. While for quality aspect, water has been sprayed to the pavement surface for an hour and with $5 \mathrm{~m}^{3} / \mathrm{min}$ inlet intensity, quantity of infiltrated water has been measured on three different intensity. Measurements have been done every 15 minutes in order to evaluate the quantity and quality of infiltrated water.

\section{Results and Discussion}

Pervious pavement has been suggested to allow percolation or infiltration of storm water through the surface into the soil below where the water is naturally filtered and pollutants are removed.

Porous asphalt is produced and placed using the same methods as conventional asphalt concrete; it differs in that fine (small) aggregates are omitted from the asphalt mixture. The remaining large, single-sized aggregate particles leave open voids that give the material its porosity and permeability. To ensure pavement strength, fiber may be added to the mix or a polymer-modified asphalt binder may be used.[10] Generally, porous asphalt pavements are designed with a subsurface reservoir that holds water that passes through the pavement, allowing it to evaporate and/or percolate slowly into the surround soils.

Permeable Concrete Blocks are blocks which has been made by concrete and there are some seams and gaps between them, runoff is able to pass through these gaps and reduce the amount of runoff.

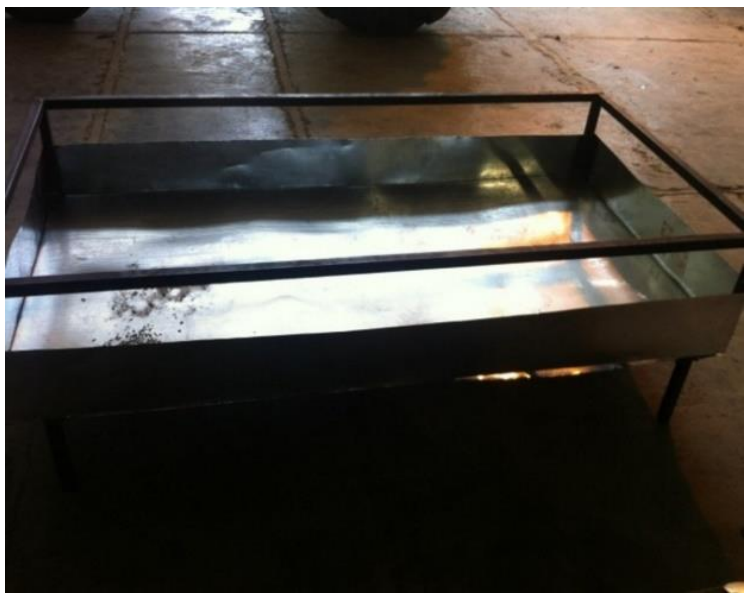

Figure 1. The experimental steel box

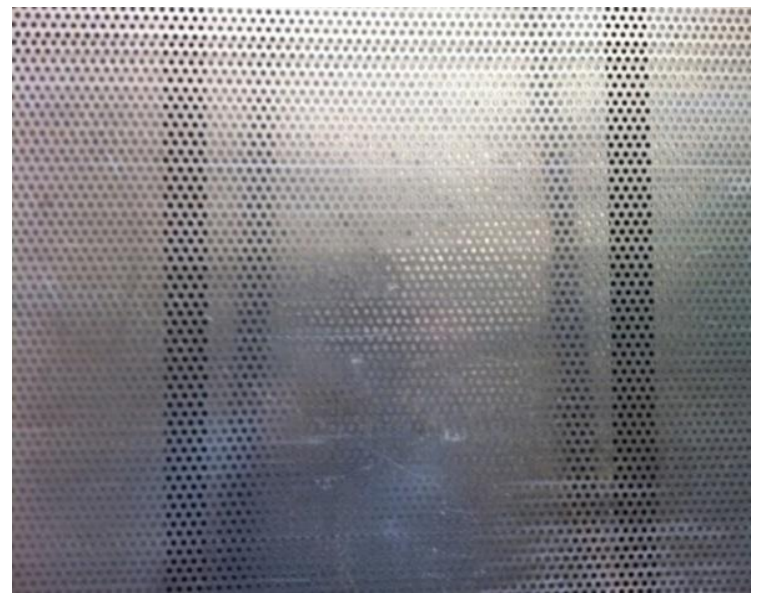

Figure 2. The bottom plate made of Galvanize 




Figure 3. The 8 setup spray

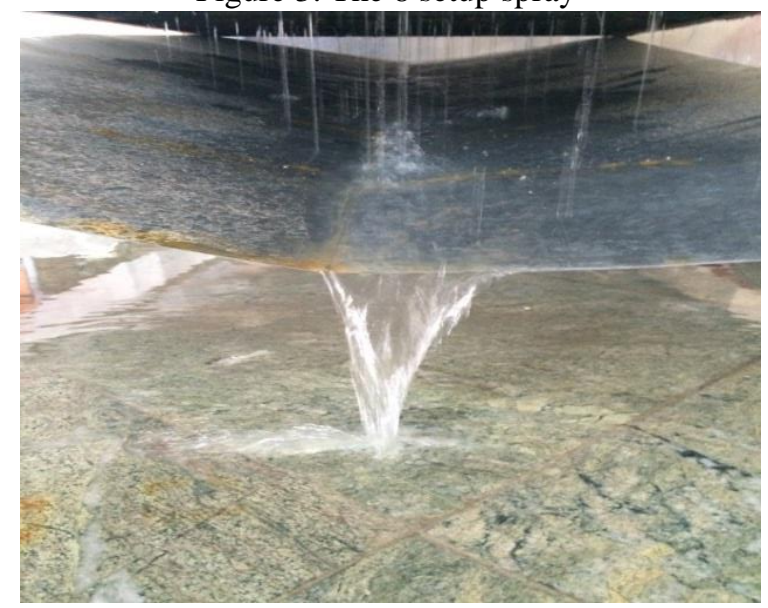

Figure 5. Infiltrated water collecting plate

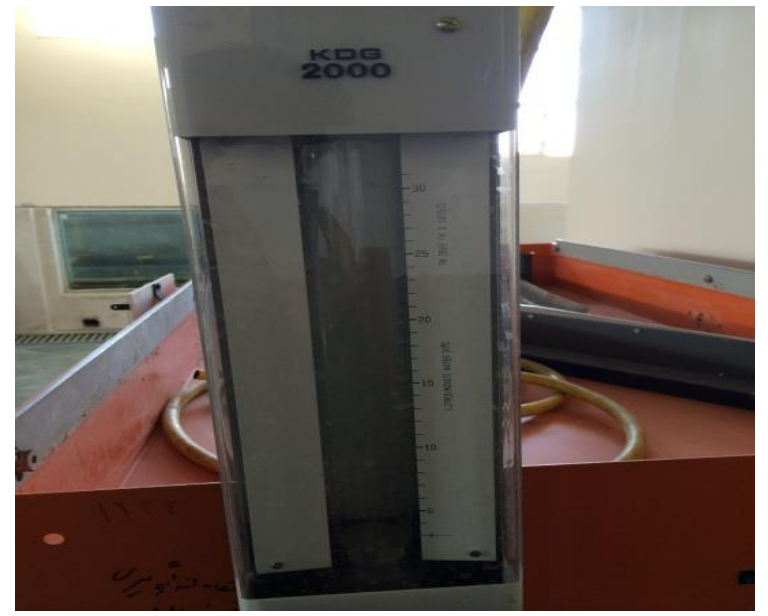

Figure 4. The flow meter apparatus

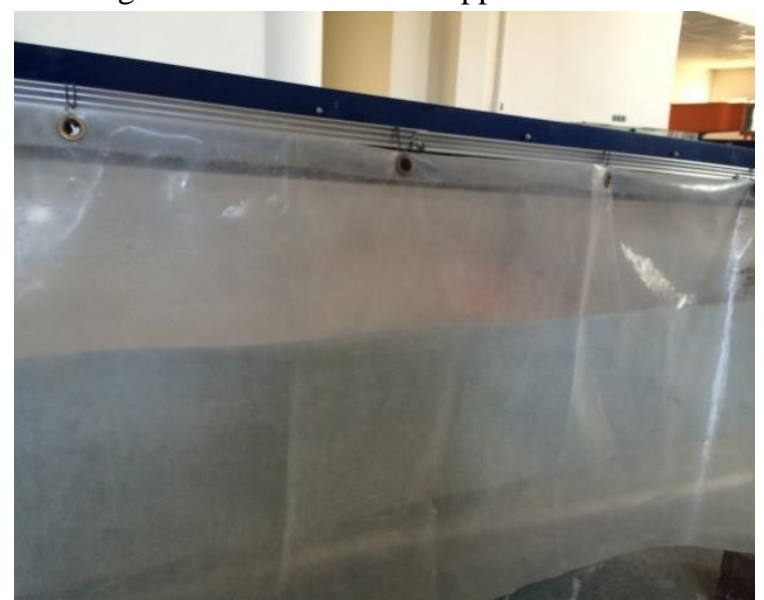

Figure 6. The plastic cover

\subsection{Water Quantity}

The methodology used to analyze the water quantity is based on comparing the volume of input water with infiltrated water through the Concrete Blocks, Travertine and Porous Asphalt under three different inlet intensity, 5, 10 and $15 \mathrm{~L} / \mathrm{min}$. (Figure 6).

As it is clear in Figure 6, Concrete Blocks has the best efficiency in passing the water, it might be because of cracks between each blocks. While porous asphalt experienced the lowest efficiency in compare to two other pavements, Travertine reduce the runoff to the acceptable amount.

Figure 6 indicates how each pavement behave based on different inlet intensities; as much as the inlet intensity increases the efficiency of pavement would decrease.

$$
\mathbf{Q}=\mathbf{5} \mathrm{L} / \mathrm{MIN}
$$

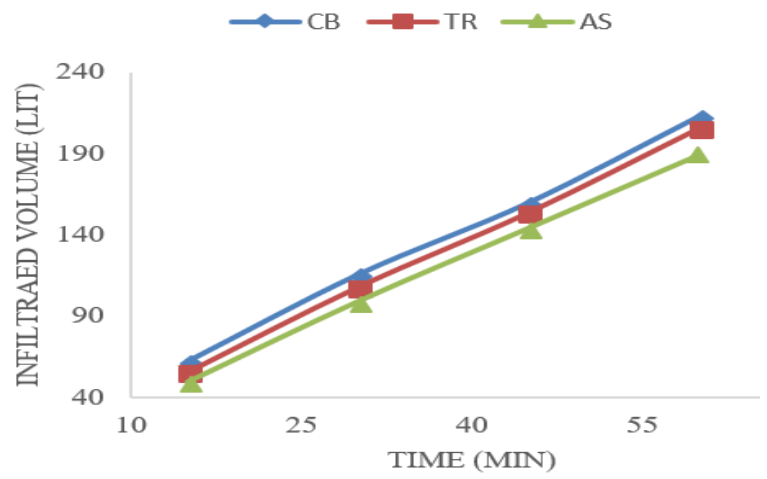

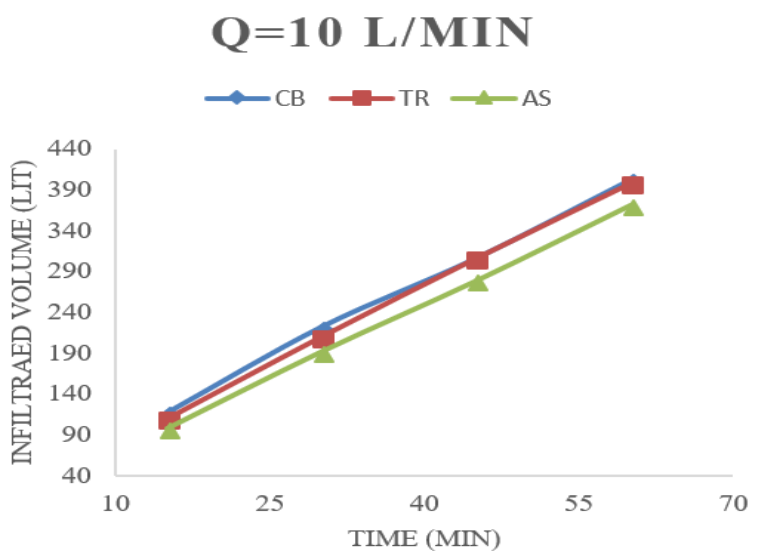




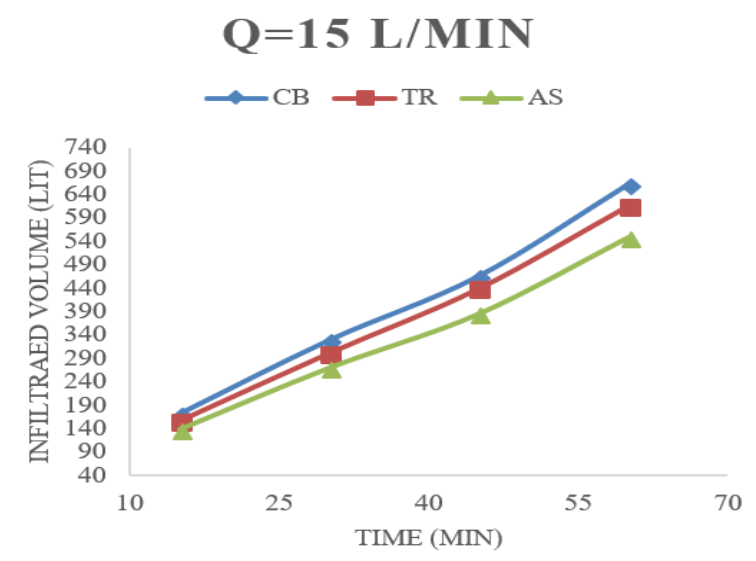

Figure 6. Input and output volumes of three different types of pavements

Figure 6 shows that the travertine pavement is able to reduce the volume of storm water up to $68.66 \%$ during 60 minutes experiment. It should also be mentioned that as same as other pervious pavements, the efficiency of travertine experienced a reduction as the inlet intensity increases from $5 \mathrm{~L} / \mathrm{min}$ to $15 \mathrm{~L} / \mathrm{min}$.

Generally, Figure 6 reveals an acceptable trend of Travertine based on two other famous types of pervious pavements.

\subsection{Water Quality}

The main aim of water quality analyses is to verify if the quality of the water passed through Travertine was good enough to be used for non-potable demands.

Water quality parameters could be selected according to different aspects. In our study which has been held in Iran, the parameters selected based on Iran non-potable Standard Water which could be considered as high quality water. Figure 7 indicates the concentration of important factors of water quality, Copper, Zinc, Lead and Cadmium, over an hour experiment.
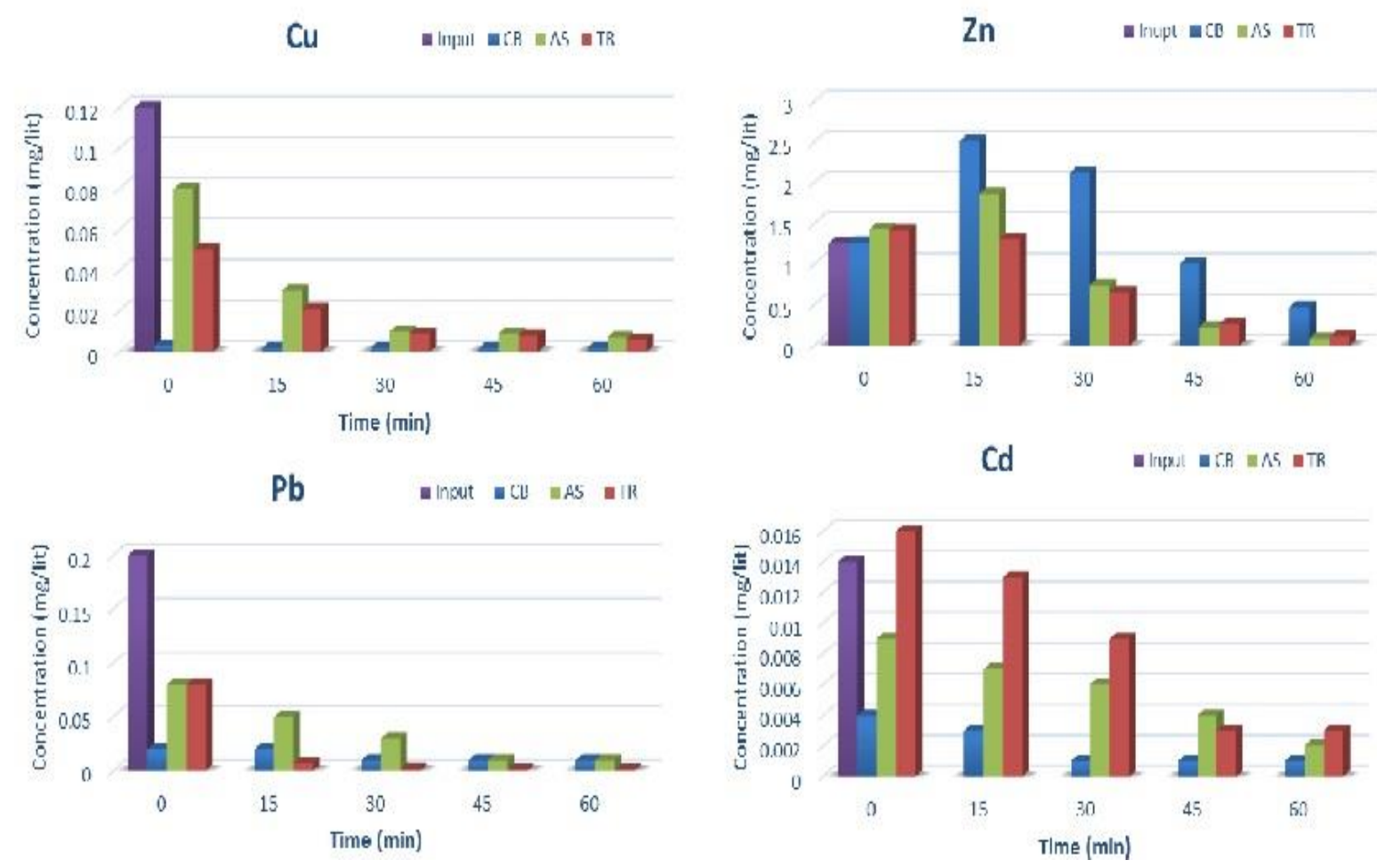

Figure 7. a - Copper, b - Zinc - Lead, d - Cadmium 
Figure 7 shows the input and output concentrations of the pollutants over an hour experiment: $95 \%$ of the Copper and up to $99 \%$ of Lead were retained on the travertine pavement, although the concentration of Zinc was more than the input concentration levels in the two first trials., which may be due to the Zinc in the galvanized metal pavement structure (Jayasuriya, 2007). The concentration of Cadmium experienced a similar fluctuation in the first trial. Such fluctuation again may be due to the travertine material and its component, which needs further investigation to examine this phenomenon.

\section{Conclusion}

The filtration characteristics of travertine pavement has been studied through quantitative and qualitative experiments. The experimental results show that regarding quantity, three pavements were differentiated with distinct behavior. Although concrete blocks have almost the best performances during an hour experiment, the Travertine pavement infiltrated more runoff than porous asphalt in all three inlet intensities.

Regarding quality, it is really hard to generalize the data to choose the best pavement but it was almost clear that in initial period of experiments rainfall washed the pavement's materials after the simulation process and it caused that the passed water quality was not enough suitable to be used in beginning of experiments.

Travertine pavement which used for the first time, could be useful material in water sensitive urban design as it helps to improve storm water quality, reduce peak flow and if properly designed and constructed, allows the infiltrated water to be reused.

Therefore, the travertine material has a great potential in many practices, particularly in the reuse of storm water.

\section{References}

Acioli, L. A., Da Silveria, A. L. L., \& Goldenfum, J. A. (2005). Experimental study of Permeable Reservoir Pavements for Surface Runoff Control at Source. 10th International Conference on Urban Drainage, Copenhagen/Denmark.

Brattebo, B. O., \& Booth, D. B. (2003). Long-term storm water quantity and quality performance of permeable pavement systems. Water Research, 37, 4369-4376. https://doi.org/10.1016/S0043-1354(03)00410-X

Castro, F. D., Bayon, R. J., Rodriguez, J., \& Ballester, F. (2005). Sistemas Urbanos de Drenaje Sostenible (SUDS). Interciencia, 30(5), 255-260.

Elena, G. U., Amaya, V. N., Joseba, R., Bayon, J., Hernandez, R. R. J., \& Daniel, C. F. (2010). Design and Construction of an Experimental Pervious Paved Parking Area to Harvest Reusable Rainwater. Novatech Conference, Lyon, France.

Gilbert, J. K., \& Clausen, J. C. (2006). Stormwater Runoff Quality and Quantity from Asphalt Paver, and Crushed Stone Driveways in Connecticut. Water Research, 40, 826-832. https://doi.org/10.1016/j.watres.2005.12.006

Hatt, B. E., Deletic, A., \& Fletcher, T. D. (2006). Integrated Treatment and Recycling of Stormwater: A Review of Australian Practice. Journal of Environmental Management, 79, 102-113. https://doi.org/10.1016/j.jenvman.2005.06.003

Jayasuriya, N., Kadurupokune, N., Othman, M., \& Jesse, K. (2007). Managing Stormwater Productively Using Pervious Pavements. Novatech Conference.

Zhang, J. (2006). Laboratory scale study of infiltration from Pervious pavement. Master thesis, School of Civil, Environmental and Chemical Engineering, Rmit University, Melbourne, Australia.

\section{Note}

Note 1. Ferdowsi University of Mashhad

\section{Copyrights}

Copyright for this article is retained by the author(s), with first publication rights granted to the journal.

This is an open-access article distributed under the terms and conditions of the Creative Commons Attribution license (http://creativecommons.org/licenses/by/4.0/). 